\title{
CANONICAL RELATIVIZED CYLINDRIC SET ALGEBRAS
}

\author{
ROGER D. MADDUX
}

(Communicated by Andreas Blass)

\begin{abstract}
For every suitable relational structure there is a canonical relativized cylindric set algebra. This construction is used to obtain a generalization of Resek's relative representation theorem, and a stronger version of the "Stone type representation theorem" by Andréka and Thompson.
\end{abstract}

\section{$\S 1$. INTRODUCTION}

Let $2 \leq n<\omega . \mathrm{MGR}_{n}$ is the set of $n$-ary merry-go-round identities:

$$
\mathrm{s}_{\kappa_{1}}^{\lambda} \mathrm{s}_{\kappa_{2}}^{\kappa_{1}} \mathrm{~s}_{\kappa_{3}}^{\kappa_{2}} \ldots \mathrm{s}_{\kappa_{n}}^{\kappa_{n-1}} \mathrm{~s}_{\lambda}^{\kappa_{n}} \mathrm{c}_{\lambda} x=\mathrm{s}_{\kappa_{n}}^{\lambda} \mathrm{s}_{\kappa_{1}}^{\kappa_{n}} \mathrm{~s}_{\kappa_{2}}^{\kappa_{1}} \ldots \mathrm{s}_{\kappa_{n-1}}^{\kappa_{n-2}} \mathrm{~s}_{\lambda}^{\kappa_{n-1}} \mathrm{c}_{\lambda} x
$$

where $\lambda, \kappa_{1}, \ldots, \kappa_{n}$ are distinct ordinals. The merry-go-round identities are defined in D. Resek's dissertation [R75]. (See [R75, pp. 2-3, and 2.3.4, p. 34], or [HMT85, 3.2.88(1)], or [HR75, pp. 382-383]. For all other unexplained terminology and notation see [HMT71] or [HMT85].) One of the most significant results of $[R 75]$ is the relative representation theorem:

Theorem $\mathbf{A}([\mathbf{R} 75,5.23])$. Suppose $2 \leq \alpha<\omega, \mathfrak{A} \in \mathrm{CA}_{\alpha}, \mathfrak{A}$ is simple, complete, atomic, and satisfies $\mathrm{MGR}_{\kappa}$ for $2 \leq \kappa<\alpha$. Then $\mathfrak{A}$ is isomorphic to a relativized cylindric set algebra, i.e., $\mathfrak{A} \in \mathrm{IRICs}_{\alpha}$.

Resek's proof of the relative representation theorem is very long, on the order of 100 typed pages. (The proof shows that $\mathfrak{A}$ must be complete, even though this is not explicitly assumed in the statement of Theorem 5.23 in [R75]. Hence "complete" should be inserted after "atomistic" in the statement of Theorem 4.3 in [HR75].)

Resek's relative representation theorem has the following consequence.

Theorem $\mathrm{B}$ ([R75, 5.27)]. For every $\alpha \geq 2$ the following are equivalent:

(i) $\mathfrak{A} \in \mathrm{CA}_{\alpha} \cap \mathrm{ISRICs}$,

(ii) $\mathfrak{A} \in \mathrm{CA}_{\alpha}$ and $\mathfrak{A}$ satisfies $\mathrm{MGR}_{\kappa}$ whenever $2 \leq \kappa<\omega$.

Theorem B shows that $\mathrm{CA}_{\alpha} \cap \mathrm{ISRICs} \mathrm{s}_{\alpha}$ is finitely axiomatizable whenever $\alpha$ is finite, and countably schematizable ([HMT85, 4.1.4]) whenever $\alpha$ is infinite.

Received by the editors March 8, 1988.

1980 Mathematics Subject Classification (1985 Revision). Primary 03G15, 20 B27. 
Resek's proof of Theorem B from Theorem A requires only six pages, but makes use of two unpublished results of L. Henkin.

R. J. Thompson has improved Theorem B in two ways. First, $\left\{\mathbf{M G R}_{n}\right.$ :

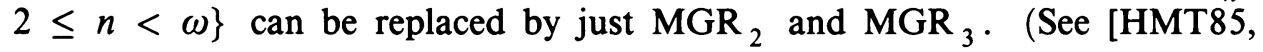
3.2.88]. $\mathrm{MGR}_{2}$ is 3.2.88(2), and $\mathrm{MGR}_{3}$ is 3.2.88(3).) Second, $\mathrm{CA}_{\alpha}$ can be replaced by the class $N A_{\alpha}$, whose definition is obtained from that of $C A_{\alpha}$ by replacing postulate $\left(\mathrm{C}_{4}\right), \mathrm{c}_{\kappa} \mathrm{c}_{\lambda} x=\mathrm{c}_{\lambda} \mathrm{c}_{\kappa} x$, of [HMT71, 1.1.1], by the weaker postulate $\left(\mathrm{C}_{4}^{*}\right), \mathrm{c}_{\kappa} \mathrm{c}_{\lambda} x \geq \mathrm{c}_{\lambda} \mathrm{c}_{\kappa} x \cdot \mathrm{d}_{\lambda \mu}$ with $\mu \neq \kappa, \lambda$ (see [AT] or [T]). With these improvements in place, Theorem $\mathrm{B}$ is called the Resek-Thompson theorem in [AT]. Thompson's proof of the Resek-Thompson theorem is similar to Resek's and is also quite long. A short proof, due to H. Andréka, is presented in [AT].

Unfortunately, much of the power of the relative representation theorem is lost in the Resek-Thompson theorem. To see this, suppose $2 \leq \alpha<\omega$ and $\mathfrak{A}$ is a simple complete atomic $\mathrm{CA}_{\alpha}$ satisfying $\mathrm{MGR}_{\kappa}$ for $2 \leq \kappa<\alpha$. Then the Resek-Thompson theorem implies that $\mathfrak{A}$ is a subalgebra of an algebra in IRICs $s_{\alpha}$, but not that $\mathfrak{A}$ is itself already in IRICs $s_{\alpha}$, as shown by the relative representation theorem. In particular, every simple finite $\mathrm{CA}_{\alpha}$ satisfying the merry-go-round identities is isomorphic to a relativized cylindric set algebra, and is not merely embeddable in such an algebra. Clearly what is needed is a short proof of Resek's relative representation theorem with Thompson's improvements. Theorem $\mathrm{C}$ below achieves this goal and more: $\alpha$ can be infinite, $\mathfrak{A}$ need not be simple, and the representation is "canonical", in the sense that no arbitrary choices are made in its construction. In particular, the axiom of choice is not used.

Theorem C. Suppose $2 \leq \alpha, \mathfrak{A} \in \mathrm{NA}_{\alpha}, \mathfrak{A}$ is complete, atomic, and satisfies $\mathrm{MGR}_{\kappa}$ for $\kappa=2,3$. Then $\mathfrak{A} \cong \mathfrak{R} \mathfrak{A} \mathfrak{A} \mathfrak{A} \in \mathrm{RICs}_{\alpha}$.

This theorem implies the Resek-Thompson theorem. For one direction it suffices to note that every algebra in ISRICs $s_{\alpha}$ satisfies MGR $_{2}$ and $\mathrm{MGR}_{3}$. For the other direction, suppose that $\mathfrak{A} \in \mathrm{NA}_{\alpha}$ and $\mathfrak{A}$ satisfies $\mathrm{MGR}_{2}$ and $\mathrm{MGR}_{3}$. Then $\mathfrak{A}$ has a complete and atomic extension $\mathfrak{A}^{\prime} \in \mathrm{NA}_{\alpha}$ which also satisfies $\mathrm{MGR}_{2}$ and $\mathrm{MGR}_{3}$, by [HMT71, 2.7.5, 2.7.13]. By Theorem C, $\mathfrak{A}^{\prime} \in \mathrm{IRICs}_{\alpha}$, so $\mathfrak{A} \in \mathrm{NA}_{\alpha} \cap \mathbf{I S R I C s _ { \alpha }}$.

The key idea in the proof of Theorem $C$ is the construction of a canonical relativized cylindric set algebra $\mathfrak{R c} \mathfrak{B}$ from any suitable relational structure $\mathfrak{B}$. (A precise definition of "suitable" is given in Definition 1 below.) For any such structure $\mathfrak{B}, \mathfrak{R} \mathfrak{B}$ is a complete and atomic relativized cylindric set algebra with unit element $V(\mathfrak{B})$ (Corollary 8) whose atoms are orbits of single sequences under a group of canonical permutations of the base $U(\mathfrak{B})$ (Lemmas 11 and 12). If $\mathfrak{A}$ is any atomic algebra which satisfies postulates $1.1 .1\left(\mathrm{C}_{0}\right)-\left(\mathrm{C}_{3}\right)$, $\left(C_{5}\right)-\left(C_{7}\right)$ of [HMT71], then the atom structure $\mathfrak{A t} \mathfrak{A}$ is suitable. There is a canonical embedding $R^{\mathfrak{A}}$ of $\mathfrak{A}$ into $\mathfrak{R} \mathfrak{A} \mathfrak{A} \mathfrak{A}$ which is a Boolean isomorphism just in case $\mathfrak{A}$ is complete. The embedding preserves the diagonal elements and 
preserves cylindrification in one direction (Lemma 17). Cylindrification is fully preserved, and the canonical embedding is therefore an isomorphism, just in case $\mathfrak{A}$ also satisfies $\left(C_{4}^{*}\right), M_{2}$, and $\mathrm{MGR}_{3}$.

Suitable structures arise naturally from certain sets of matrices of atoms of weakly associative relation algebras (defined in [M82]). If $\mathfrak{A}$ is an atomic weakly associative relation algebra, then $\mathfrak{A t C a}_{\mathfrak{t}} B_{3} \mathfrak{A}$ happens to be a suitable structure. (See [M89] for $\mathfrak{C a}$ and $B_{3}$.) The two-dimensional projection $R$ of the ternary relation $V\left(\mathfrak{A t C a} B_{3} \mathfrak{A}\right)$ was used in the first (unpublished) proof of Theorem 5.20 of [M82]. That proof did not appear in [M82] due to the difficulties encountered in trying to formulate a precise mathematical description of $R$. This paper finally overcomes those difficulties. (Also, the proof which does appear in [M82] yields a useful auxiliary result, namely Theorem 5.19.) I would like to thank Richard L. Kramer for many useful discussions in the early 1980s about $R$ and how to describe it, and the referee for extensive help in clarifying the arguments and exposition of this paper.

\section{§2. Definition of $\mathfrak{R c} \mathfrak{B}$}

Definition 1. $\mathfrak{B}$ is a suitable structure if $\mathfrak{B}=\left\langle B, T_{\kappa}, E_{\kappa \lambda}\right\rangle_{\kappa, \lambda<\alpha}$ where $\alpha$ is a nonzero ordinal, $T_{\kappa} \subseteq B \times B, E_{\kappa \lambda} \subseteq B$, and the following conditions hold for all $\kappa, \lambda, \mu<\alpha$ :

(i) $T_{\kappa}$ is an equivalence relation on $B$,

(ii) $E_{\kappa \kappa}=B$,

(iii) $E_{\kappa \lambda}=T_{\mu}^{*}\left(E_{\kappa \mu} \cap E_{\mu \lambda}\right)$ whenever $\kappa, \lambda \neq \mu$,

(iv) $T_{\kappa} \cap\left(E_{\kappa \lambda} \times E_{\kappa \lambda}\right) \subseteq I d$ whenever $\kappa \neq \lambda$.

Throughout this section we assume that $\mathfrak{B}$ is a suitable structure and $\alpha>0$.

Lemma 2. For all $\kappa, \lambda, \mu<\alpha$,

(i) $E_{\kappa \lambda}=T_{\mu}^{*} E_{\kappa \lambda}$ if $\mu \neq \kappa, \lambda$,

(ii) $B=T_{\kappa}^{*} E_{\kappa \lambda}$,

(iii) $E_{\kappa \mu} \cap E_{\mu \lambda} \subseteq E_{\kappa \lambda}$,

(iv) $E_{\kappa \lambda}=E_{\lambda \kappa}$.

Proof. Parts (i)-(iii) are immediate consequences of Definition 1(i)-(iii). For part (iv), we may assume $\kappa \neq \lambda$. By Definition 1(i)-(iii) we have $E_{\kappa \lambda} \sim E_{\lambda \kappa} \subseteq$ $T_{\kappa}^{*}\left(E_{\kappa \lambda} \sim E_{\lambda \kappa}\right) \cap T_{\kappa}^{*}\left(E_{\kappa \lambda} \cap E_{\lambda \kappa}\right)$. But $T_{\kappa}^{*}\left(E_{\kappa \lambda} \sim E_{\lambda \kappa}\right) \cap T_{\kappa}^{*}\left(E_{\kappa \lambda} \cap E_{\lambda \kappa}\right)=\varnothing$ by Definition 1(i)(iv), so $E_{\kappa \lambda} \subseteq E_{\lambda \kappa}$.

Definition 3. Let

$$
\begin{aligned}
\operatorname{Tr}(\mathfrak{B})=\bigcup_{n<\omega}\left\{\left\langle x_{0}, \kappa_{0}, \ldots, x_{n}, \kappa_{n}\right\rangle \in \in^{(n+1)}(B \times \alpha):\right. & \\
& \left.(\forall i<n)\left(x_{i} \neq x_{i+1} \wedge x_{i} T_{\kappa_{i}} x_{i+1}\right)\right\} .
\end{aligned}
$$

The sequences in $\operatorname{Tr}(\mathfrak{B})$ are called trails of $\mathfrak{B}$, or $\mathfrak{B}$-trails. Let $t=\left\langle x_{0}, \kappa_{0}\right.$, $\left.\ldots, x_{n}, \kappa_{n}\right\rangle$ be a trail of $\mathfrak{B}$. We say that $t$ begins at $x_{0}, t$ ends at $x_{n}, \kappa_{n}$ is 
the pointer of $t$, and $t$ has length $|t|=n+1$. The trail $t$ is reduced if the following conditions hold:

(i) if $1=|t|$ and $x_{0} \in E_{\kappa_{0} \lambda}$, then $\kappa_{0} \leq \lambda<\alpha$,

(ii) if $1<|t|$ then $\kappa_{n-1}=\kappa_{n}$ and for all $\lambda<\alpha, x_{n} \in E_{\kappa_{n} \lambda}$ iff $\kappa_{n}=\lambda$,

(iii) if $0 \leq i<|t|-2$, then either $x_{i} \neq x_{i+2}$ or $\kappa_{i} \neq \kappa_{i+1}$.

Finally, for every $\lambda<\alpha$, let $t \lambda=\left\langle x_{0}, \kappa_{0}, \ldots, x_{n-1}, \kappa_{n-1}, x_{n}, \lambda\right\rangle$.

All trails have length 1 or more. Condition (iii) applies only to trails of length 3 or more, and states that a reduced trail cannot have a subsequence of the form $\langle x, \lambda, y, \lambda, x\rangle$. If $t$ is a trail, then so is $t \lambda$.

Definition 4. Let $Q$ be the smallest equivalence relation on $\operatorname{Tr}(\mathfrak{B})$ which contains all pairs of $\mathfrak{B}$-trails of the following three types:

(1) $\left\langle\left\langle x_{0}, \kappa_{0}, \ldots, x_{i}, \lambda, y, \lambda, x_{i}, \kappa_{i}, \ldots, x_{n}, \kappa_{n}\right\rangle\right.$, $\left.\left\langle x_{0}, \kappa_{0}, \ldots, x_{i}, \kappa_{i}, \ldots, x_{n}, \kappa_{n}\right\rangle\right\rangle \quad$ where $0 \leq i \leq n$,

(2) $\left\langle\left\langle x_{0}, \kappa_{0}, \ldots, x_{n}, \lambda, y, \kappa_{n}\right\rangle,\left\langle x_{0}, \kappa_{0}, \ldots, x_{n}, \kappa_{n}\right\rangle\right\rangle$ where $\lambda \neq \kappa_{n}$,

(3) $\left\langle\left\langle x_{0}, \kappa_{0}, \ldots, x_{n}, \lambda\right\rangle,\left\langle x_{0}, \kappa_{0}, \ldots, x_{n}, \kappa_{n}\right\rangle\right\rangle$ where $x_{n} \in E_{\lambda \kappa_{n}}$.

For each $t \in \operatorname{Tr}(\mathfrak{B})$, let $t^{\mathfrak{B}}$ be the $Q$-class of $t$, i.e., $t^{\mathfrak{B}}=\left\{t^{\prime}: t Q t^{\prime}\right\}$. Let $U(\mathfrak{B})=\left\{t^{\mathfrak{B}}: t \in \operatorname{Tr}(\mathfrak{B})\right\} . U(\mathfrak{B})$ is called the canonical base for $\mathfrak{B}$, or $\mathfrak{B}$-base, and the equivalence classes in $U(\mathfrak{B})$ are called canonical base points.

The relations used to define $Q$ are "reductions". A reduction of type (1) consists of the replacement of any subsequence of the form $\langle x, \lambda, y, \lambda, x\rangle$ by $\langle x\rangle$. Equivalent trails have the same beginnings, but may have different ends and different pointers, due to reductions of type (2) and (3).

The intuition behind trails and the definition of $Q$ arises in the following way. Suppose that $\mathfrak{B}$ is a suitable structure and $\mathfrak{B}$ is already represented by a relativized cylindric set algebra with base $U$. Refer to the elements of $U$ as "base points". Suppose also that $R_{x}$ is the set of sequences in ${ }^{\alpha} U$ associated with each $x \in B$. This representation must have certain properties. First, if $x \in E_{\kappa \lambda}$ then the $\kappa$-term of any sequence associated with $x$ must be the same as the $\lambda$-term of that sequence, or, briefly, $p_{\kappa}=p_{\lambda}$ for every $p \in R_{x}$. Second, if $x T_{\kappa} y$, then every sequence $p \in R_{x}$ can have its $\kappa$-term altered to obtain a sequence $p^{\prime} \in R_{y}$, hence $p_{\mu}^{\prime}=p_{\mu}$ whenever $\mu \neq \kappa$. We wish to describe the representation $R$ of $\mathfrak{B}$ using trails, and then use that description to construct a canonical representation entirely in terms of $\mathfrak{B}$-trails.

Let $t=\langle x, \kappa, \ldots\rangle$ be a trail beginning at $x$. Then $t$ may be conceived as instructions which can be applied to any sequence $p \in{ }^{\alpha} U$ associated with $x$ (but not applicable to any sequence not associated with $x$ ). The instructions in $t$ select a particular base point, depending on $p$. It will turn out that $t$ is reduced if there is no shorter trail which leads from $p$ to the base point selected by $t$.

Some examples will show how the instructions should be followed. Let $p \in$ $R_{x}$. The trail $\langle x, \kappa\rangle$ says, "Select the $\kappa$-term of the sequence." So, following 
$\langle x, \kappa\rangle$ from $p$ leads to the base point $p_{\kappa}$. The trail $\langle x, \kappa, y, \lambda\rangle$ says, "Alter the $\kappa$-term of the sequence to get a sequence associated with $y$, and then select the $\lambda$-term of the latter sequence." Following $\langle x, \kappa, y, \lambda\rangle$ from $p$ leads to the base point $p_{\lambda}^{\prime}$ for some $p^{\prime} \in R_{y}$ such that $p_{\mu}=p_{\mu}^{\prime}$ whenever $\mu \neq \kappa$. Finally, the trail $\langle x, \kappa, y, \lambda, z, \nu\rangle$ says, "Alter the $\kappa$-term of the first sequence to get a sequence associated with $y$, then alter the $\lambda$-term of the latter sequence to get a third sequence associated with $z$, and then select the $\nu$-term of the third sequence."

These examples show that the pointer has a different role from the other ordinals in a trail. The pointer tells which base point in the final sequence to select, while the other ordinals in the trail tell which term in one intermediate sequence should be altered to get the next sequence.

The instructions may be ambiguous. There may be more than one way to alter a sequence to get a satisfactory new sequence. In could happen, for example, that $p \in R_{x}, x T_{\kappa} y, p^{\prime}, p^{\prime \prime} \in R_{y}, p_{\mu}=p_{\mu}^{\prime}=p_{\mu}^{\prime \prime}$ whenever $\mu \neq \kappa$, and yet $p^{\prime} \neq p^{\prime \prime}$. Following $\langle x, \kappa, y, \lambda\rangle$ from $p$ could therefore lead to either $p_{\lambda}^{\prime}$ or $p_{\lambda}^{\prime \prime}$. We could, of course, make the instructions unambiguous by well-ordering the elements of $U$ and always choosing the first suitable base point as the new $\kappa$-term. We could also complicate the structure of trails by adding ordinals to index which base point is being selected at each successive alteration. This procedure would considerably complicate the definition of $\mathfrak{R} \mathfrak{B}$. Nevertheless, the main result could be proved using such a construction.

Instead we assume that the representation has a special property which guarantees the instructions in every trail are unambiguous. More precisely, we assume that if $x, y \in \mathfrak{B}, p \in R_{x}, x T_{\kappa} y, p^{\prime}, p^{\prime \prime} \in R_{y}$, and $p_{\mu}=p_{\mu}^{\prime}=p_{\mu}^{\prime \prime}$ whenever $\mu \neq \kappa$, then $p^{\prime}=p^{\prime \prime}$. We will derive the definition of $Q$ under this assumption of unambiguity.

Consider the base points which can be reached from $p \in R_{x}$ by following trails which begin at $x$. Different trails may lead to the same base point. The definition of the equivalence relation $Q$ is designed so that if two trails are equivalent via $Q$, then they lead to the same base point. This is why canonical base points are defined as $Q$-equivalence classes. Depending on the structure of the given representation, it may or may not be true that trails leading to the same base point are equivalent, but this will be true in the canonical representation.

To see the need for each of the three types of reductions in $Q$, consider the following example. Suppose $p \in R_{x}, x \neq y, p^{\prime} \in R_{y}, x T_{0} y, x, y \in$ $E_{23}, p_{\mu}=p_{\mu}^{\prime}$ whenever $\mu \neq 0$, and $p_{0}, p_{1}, p_{2}, p_{0}^{\prime}$ are distinct. Following $\langle x, 0\rangle$ from $p$ leads to $p_{0}$, while following $\langle x, 0, y, 0\rangle$ from $p$ leads to $p_{0}^{\prime}$, a base point distinct from $p_{0}$. On the other hand, following either $\langle x, 1\rangle$ or $\langle x, 0, y, 1\rangle$ from $p$ leads to the same base point, since $p_{1}=p_{1}^{\prime}$ and, by the assumption of unambiguity, there can be no sequence in $R_{y}$ other than $p^{\prime}$ which coincides with $p$ at all terms except the 0 -term. We must therefore 
define $Q$ so that $\langle x, 1\rangle Q\langle x, 0, y, 1\rangle$. This justifies the inclusion of reductions of type (2) in $Q$. Following either $\langle x, 2\rangle$ or $\langle x, 3\rangle$ from $p$ also leads to the same base point, since $x \in E_{23}$ and hence $p_{2}=p_{3}$. By including reductions of type (1) in the definition of $Q$ we accordingly get $\langle x, 2\rangle Q\langle x, 3\rangle$. For any $\kappa$, following $\langle x, 0, y, 0, x, \kappa\rangle$ from $p$ requires first moving from $p$ to $p^{\prime}$ (no other choice is available, by unambiguity), and then from $p^{\prime}$ to a sequence in $R_{x}$ which differs from $p^{\prime}$ only in its 0 -term. But there already is such a sequence, namely $p$. Hence, by unambiguity, the last sequence obtained by following $\langle x, 0, y, 0, x, \kappa\rangle$ from $p$ must be $p$ itself and the selected base point must be $p_{\kappa}$. Since this point is also selected by $\langle x, \kappa\rangle$, we must define $Q$ so that $\langle x, 0, y, 0, x, \kappa\rangle Q\langle x, \kappa\rangle$. This is accomplished by including reductions of type (1). It turns out that no other reductions are needed. Even reductions of type (1) are not strictly necessary to construct a relative representation of a suitable structure. By including reductions of type (1) we guarantee that the resulting canonical relative representation is, in fact, unambiguous. Note that the reduced trails in this example are $\langle x, 0\rangle,\langle x, 1\rangle,\langle x, 2\rangle$, and $\langle x, 0, y, 0\rangle$, which lead to $p_{0}, p_{1}, p_{2}$, and $p_{0}^{\prime}$, respectively.

In the next definition we give short names to the reductions and certain other relations. Then in the lemma we show that every canonical base point contains a unique reduced trail, and give an algorithm for computing the unique reduced trail in $t^{\mathfrak{B}}$ from $t$.

For any binary relation $X$, the transitive closure of $X$ is $X^{\omega}=X \cup X^{2} \cup$ $X^{3} \cup \ldots$, where $X^{2}=X\left|X, X^{3}=X\right| X \mid X$, etc., and $D o X$ is the domain of $X$. For any set $U, I d_{U}$ is the identity relation on $U$.

Definition 5. For $n=1,2,3$, let $P_{n}$ be the set of pairs of $\mathfrak{B}$-trails of type $(n)$ in Definition 4. Let $P_{4}=P_{3} \cap\{\langle t \kappa, t \lambda\rangle: \kappa>\lambda\}, P_{5}=P_{3}\left|\left(P_{1} \cup P_{2}\right)\right| P_{3}$, $P_{6}=P_{3} \cup P_{5}, Z=\operatorname{Tr}(\mathfrak{B}) \sim D o\left(P_{4} \cup P_{5}\right)$, and $P_{7}=P_{6}^{\omega} \mid I d_{Z}$.

\section{Lemma 6.}

(i) $P_{2}$ is a function, i.e., $P_{2}^{-1} \mid P_{2} \subseteq I d_{\operatorname{Tr}(\mathfrak{B})}$,

(ii) $P_{3}$ is an equivalence relation on $\operatorname{Tr}(\mathfrak{B})$,

(iii) $P_{1}^{-1}\left|P_{1}=I d_{\operatorname{Tr}(\mathfrak{B})} \cup P_{1}\right| P_{1}^{-1}$,

(iv) $P_{2}^{-1}\left|P_{1}=P_{2} \cup P_{1}\right| P_{2}^{-1}$ and $P_{1}^{-1}\left|P_{2}=P_{2}^{-1} \cup P_{2}\right| P_{1}^{-1}$,

(v) $P_{1}^{-1}\left|P_{3}=P_{3}\right| P_{1}^{-1}$ and $P_{3}\left|P_{1}=P_{1}\right| P_{3}$,

(vi) $P_{1}^{-1}\left|P_{3}\right| P_{1} \subseteq P_{5} \mid P_{5}^{-1} \cup P_{3}$,

(vii) $P_{1}^{-1}\left|P_{3}\right| P_{2} \subseteq P_{5} \mid P_{5}^{-1} \cup P_{5}^{-1}$ and $P_{2}^{-1}\left|P_{3}\right| P_{1} \subseteq P_{5} \mid P_{5}^{-1} \cup P_{5}$,

(viii) $P_{2}^{-1}\left|P_{3}\right| P_{2} \subseteq P_{3}$,

(ix) $P_{6}^{-1}\left|P_{6} \subseteq P_{6}\right| P_{6}^{-1}$,

(x) $Q=\left(P_{6} \cup P_{6}^{-1}\right)^{\omega}=P_{6}^{\omega} \mid\left(P_{6}^{-1}\right)^{\omega}$,

(xi) for every $t \in \operatorname{Tr}(\mathfrak{B}), t$ is reduced iff $t \in Z$,

(xii) $Q=P_{6}^{\omega}\left|I d_{Z}\right|\left(P_{6}^{-1}\right)^{\omega}=P_{7} \mid P_{7}^{-1}$,

(xiii) $I d_{Z} \mid P_{7}=I d_{Z}$,

(xiv) $P_{7}$ is a function. 
Proof. Parts (i), (iii)-(v) follow just from the relevant parts of Definition 4. Part (ii) follows from Definition 1(ii) and Lemma 2(iii)(iv). Part (vi) follows from (ii), (iii), and (v). Part (vii) follows from (ii), (iv), and (v). Part (viii) follows from Definition 4 and Lemma 2(i). Parts (ii), (vi)-(viii) imply (ix).

We have $I d_{\operatorname{Tr}(\mathfrak{B})} \subseteq P_{1} \cup P_{2} \cup P_{3} \subseteq P_{6} \subseteq Q$ by part (ii), and $Q$ is the equivalence relation generated by $P_{1} \cup P_{2} \cup P_{3}$, so $Q=\left(P_{6} \cup P_{6}^{-1}\right)^{\omega}$. Part (ix) implies $\left(P_{6} \cup P_{6}^{-1}\right)^{\omega} \subseteq P_{6}^{\omega} \mid\left(P_{6}^{-1}\right)^{\omega}$ by induction, and the opposite inclusion is trivially true. Thus $(x)$ holds.

If $|t|=1$ then $t$ is reduced iff $t \notin D o P_{4}$. If $|t|>1$ then $t$ is reduced iff $t \notin D o P_{5}$. This follows from the observation that if the pointer can be changed by a type (3) reduction, then a type (2) reduction can be performed. More precisely, if $|t|>1, t P_{3} t^{\prime}$, and $t \neq t^{\prime}$, then $t^{\prime}=t \lambda$ for some $\lambda$, and hence either $t \in D o P_{2}$ or $t^{\prime} \in D o P_{2}$. Thus (xi) holds.

Let $R=P_{4} \cup P_{5}$. Then $\operatorname{Tr}(\mathfrak{B})=Z \cup D o R$, so

$$
\begin{aligned}
I d_{\operatorname{Tr}(\mathfrak{B})} & =I d_{Z} \cup I d_{D o R} \subseteq I d_{Z} \cup R \mid R^{-1} \\
& =I d_{Z} \cup R\left|I d_{T r(\mathfrak{B})}\right| R^{-1} \subseteq I d_{Z} \cup R\left|\left(I d_{Z} \cup R \mid R^{-1}\right)\right| R^{-1} \\
& =I d_{Z} \cup R\left|I d_{Z}\right| R^{-1} \cup R^{2} \mid\left(R^{-1}\right)^{2} .
\end{aligned}
$$

Continuing in this way, we get

$$
I d_{T r(\mathfrak{B})}=I d_{Z} \cup \bigcup_{0<k<n} R^{k}\left|I d_{Z}\right|\left(R^{-1}\right)^{k} \cup R^{n} \mid\left(R^{-1}\right)^{n}
$$

whenever $2 \leq n<\omega$. If $t P_{5} t^{\prime}$ then $|t|>\left|t^{\prime}\right|$, and if $t \kappa P_{4} t \lambda$ then $\kappa>\lambda$, so there are no infinite $R$-chains. Therefore, given an arbitrary $t \in \operatorname{Tr}(\mathfrak{B})$, there is some $n$ such that $\langle t, t\rangle \notin R^{n} \mid\left(R^{-1}\right)^{n}$ and $2 \leq n<\omega$, which implies, by (1), that $\langle t, t\rangle \in I d_{Z} \cup \bigcup_{0<k<n} R^{k}\left|I d_{Z}\right|\left(R^{-1}\right)^{k}$. This proves $I d_{T r(\mathfrak{B})} \subseteq$ $I d_{Z} \cup R^{\omega}\left|I d_{Z}\right|\left(R^{-1}\right)^{\omega}$. Note that $I d_{Z} \cup R \subseteq P_{6}$. Consequently $I d_{\operatorname{Tr}(\mathfrak{B})} \subseteq$ $P_{6}^{\omega}\left|I d_{Z}\right|\left(P_{6}^{-1}\right)^{\omega}$ and $P_{6}^{\omega} \mid P_{6}^{\omega}=P_{6}^{\omega}$. By $(\mathrm{x}), \quad Q=P_{6}^{\omega}\left|I d_{\operatorname{Tr}(\mathfrak{B})}\right|\left(P_{6}^{-1}\right)^{\omega} \subseteq$ $P_{6}^{\omega}\left|P_{6}^{\omega}\right| I d_{Z}\left|\left(P_{6}^{-1}\right)^{\omega}\right|\left(P_{6}^{-1}\right)^{\omega}=P_{6}^{\omega}\left|I d_{Z}\right|\left(P_{6}^{-1}\right)^{\omega}$. Note that $P_{7}^{-1}=I d_{Z} \mid\left(P_{6}^{\omega}\right)^{-1}=$ $I d_{Z} \mid\left(P_{6}^{-1}\right)^{\omega}$, so $Q=P_{7} \mid P_{7}^{-1}$. Thus (xii) holds.

By the relevant definitions, $I d_{Z}\left|P_{6}=I d_{Z}\right|\left(I d_{T r(\mathfrak{B})} \cup P_{4} \cup P_{4}^{-1} \cup P_{5}\right)=I d_{Z} \cup$ $I d_{Z} \mid P_{4}^{-1}$. Also, $P_{4}^{-1}\left|P_{6} \subseteq P_{3}\right| P_{6}=P_{6}$ by part (ii). Consequently $I d_{Z} \mid P_{6}^{\omega}=$ $I d_{Z} \cup I d_{Z} \mid P_{4}^{-1}$ by induction. Also, $P_{4}^{-1} \mid I d_{Z}=\varnothing$, so $I d_{Z}\left|P_{7}=I d_{Z}\right| P_{6}^{\omega} \mid I d_{Z}=$ $\left(I d_{Z} \cup I d_{Z} \mid P_{4}^{-1}\right) \mid I d_{Z}=I d_{Z}$. Thus (xiii) holds.

By the definition of $P_{7},(x)$, (xii), and (xiii), $P_{7}^{-1}\left|P_{7}=I d_{Z}\right|\left(P_{6}^{\omega}\right)^{-1}\left|P_{6}^{\omega}\right| I d_{Z} \subseteq$ $I d_{Z}|Q| I d_{Z}=I d_{Z}\left|P_{7}\right| P_{7}^{-1} \mid I d_{Z}=I d_{Z}$, so (xiv) holds.

By Lemma $6, P_{7}$ is a function contained in $Q, P_{7}(t)$ is reduced, and $t$ is reduced just in case $P_{7}(t)=t$. Thus $P_{7}$ maps each trail $t$ to the unique reduced trail in $t^{\mathfrak{B}}$. Since $P_{3}$ is an equivalence relation and $P_{3}\left|P_{5}=P_{5}=P_{5}\right| P_{3}$, 
we have $P_{6}^{\omega}=\left(P_{3} \cup P_{5}\right)^{\omega}=P_{3} \cup P_{5}^{\omega}$, so $P_{7}=P_{6}^{\omega}\left|I d_{Z}=\left(P_{3} \cup P_{5}^{\omega}\right)\right| I d_{Z}=$ $\left(I d_{T r(\mathfrak{B})} \cup P_{4} \cup P_{4}^{-1} \cup P_{5}^{\omega}\right)\left|I d_{Z}=I d_{Z} \cup\left(P_{4} \cup P_{5}^{\omega}\right)\right| I d_{Z}$. In other words, to compute $P_{7}(t)$ in case $t$ is not reduced, follow a $P_{5}$-chain until a trail is obtained which is not in $D o P_{5}$, and then reduce the pointer as much as possible by using $P_{4}$ once. Notice that $\left|P_{7}(t)\right| \leq|t|$ for every trail $t$. If $|t|=1$, then $t \notin D o P_{5}$, and either $t$ is already reduced, or else $t P_{4} P_{7}(t)$, where $P_{7}(t)=t \kappa$ for some ordinal $\kappa$ which is smaller than the pointer of $t$.

Definition 7. For every $x \in B, \mathrm{R}_{x}^{\mathfrak{B}}=\left\{\left\langle(t \kappa)^{\mathfrak{B}}: \kappa<\alpha\right\rangle: t \in \operatorname{Tr}(\mathfrak{B}), t\right.$ ends at $x\}$. Let $V(\mathfrak{B})=\bigcup_{x \in B} \mathrm{R}_{x}^{\mathfrak{B}}$ and $\mathfrak{R} \mathfrak{B}=\mathfrak{R l}_{V(\mathfrak{B})} \mathfrak{A}$, where $\mathfrak{A}$ is the subalgebra of $\mathfrak{S b}^{\alpha} U(\mathfrak{B})$ which is completely generated by $\left\{R_{x}^{\mathfrak{B}}: x \in B\right\} . \mathfrak{R c} \mathfrak{B}$ is the canonical relativized cylindric set algebra of $\mathfrak{B}$.

Corollary 8. $\mathfrak{R c} \mathfrak{B}$ is a complete atomic relativized cylindric set algebra.

It turns out that $\mathrm{R}_{x}^{\mathfrak{B}}$ is an atom in $\mathfrak{R c} \mathfrak{B}$, for every $x \in B$. To prove this we will use the next lemma.

For every set $U$, if $p$ is a one-to-one function from $U$ into $U$, then $\tilde{p}$ is the function which maps $S b\left({ }^{\alpha} U\right)$ to $S b\left({ }^{\alpha} U\right)$ and is defined by $\tilde{p} X=\{a \in$ $\left.{ }^{\alpha} U: a \mid p^{-1} \in X\right\}$ for every $X \subseteq{ }^{\alpha} U$. (See [HMT85, p. 15].) It follows that if $p$ is a permutation of $U$, then $\tilde{p} X=\{a \mid p: a \in X\}$.

Lemma 9. Suppose $U$ and $B$ are sets, $R_{x} \subseteq{ }^{\alpha} U$ for every $x \in B$, and $P$ is a set of permutations of $U$. Assume, for every $x \in B$, that $P$ preserves $R_{x}$, i.e., $(\forall p \in P)\left(\tilde{p} R_{x}=R_{x}\right)$, and that $P$ acts transitively on $R_{x}$, i.e., $(\forall a, b \in$ $\left.R_{x}\right)(\exists p \in P)(a \mid p=b)$. Let $\mathfrak{A}$ be the subalgebra of $\mathfrak{S b}^{\alpha} U$ which is completely generated by $\left\{R_{x}: x \in B\right\}$. Then $\left\{R_{x}: x \in B\right\} \subseteq$ At $\mathfrak{A}$.

Proof. $P$ preserves $\left\{R_{x}: x \in B\right\}$, and therefore preserves all relations $S$ in $\mathfrak{A}$. If some such relation $S$ were properly contained within some $R_{x}$, then it would be possible, by the transitivity of $P$ on $R_{x}$, to pick a $p \in P$ which moves some sequence from $S$ to $R_{x} \sim S$, contradicting the fact that every $p \in P$ must preserve $S$.

Definition 10. For every $\mathfrak{B}$-trail $s=\left\langle x_{0}, \kappa_{0}, x_{1}, \kappa_{1}, \ldots, x_{n-1}, \kappa_{n-1}, x_{n}, \kappa_{n}\right\rangle$ let $\check{s}=\left\langle x_{n}, \kappa_{n-1}, x_{n-1}, \kappa_{n-2}, \ldots, x_{1}, \kappa_{0}, x_{0}, \kappa_{n}\right\rangle$. If $t=\left\langle y_{0}, \lambda_{0}, \ldots, y_{m}, \lambda_{m}\right\rangle$ is any other $\mathfrak{B}$-trail, then $s \odot t$ is defined if $s$ ends where $t$ begins, in which case

$$
s \odot t=\left\langle x_{0}, \kappa_{0}, x_{1}, \kappa_{1}, \ldots, x_{n-1}, \kappa_{n-1}, y_{0}, \lambda_{0}, \ldots, y_{m}, \lambda_{m}\right\rangle .
$$

Also,

$$
L_{s}(t)= \begin{cases}s \odot t & \text { if } x_{n}=y_{0} \\ \check{s} \odot t & \text { if } x_{n} \neq y_{0}=x_{0} \\ t & \text { if } x_{n} \neq y_{0} \neq x_{0}\end{cases}
$$

and, for any $X \subseteq \operatorname{Tr}(\mathfrak{B}), l_{s}(X)=\bigcup_{t \in X} L_{s}(t)^{\mathfrak{B}}$. Let

$$
\operatorname{Pm}(\mathfrak{B})=\left\{l_{s}: s \in \operatorname{Tr}(\mathfrak{B})\right\} .
$$


If $s$ and $t$ are $\mathfrak{B}$-trails, then $\check{s}$ is a $\mathfrak{B}$-trail of the same length, and if $s \odot t$ is defined, then $s \odot t$ is also a $\mathfrak{B}$-trail with $|s \odot t|=|s|+|t|-1$. The associative law for $\odot$ holds whenever both sides are defined. If $|s|=1$, then $\check{s}=s$ and $L_{s}(t)=t$ for every $\mathfrak{B}$-trail $t$. Note that $(s \odot t) \kappa=s \odot t \kappa$ and $L_{s}(t) \kappa=L_{s}(t \kappa)$.

Lemma 11. Suppose $s, t \in \operatorname{Tr}(\mathfrak{B})$. Then

(i) $l_{s}\left(t^{\mathfrak{B}}\right)=L_{s}(t)^{\mathfrak{B}}$,

(ii) $t^{\mathfrak{B}}=l_{\dot{s}} l_{s}\left(t^{\mathfrak{B}}\right)=l_{s} l_{\dot{s}}\left(t^{\mathfrak{B}}\right)$,

(iii) $l_{s}$ is a permutation of $U(\mathfrak{B})$ and $\left(l_{s}\right)^{-1}=l_{\xi}$.

Proof. Suppose $s$ begins at $x_{0}$ and ends at $x_{n}$. If $t$ begins at $y_{0}$ then $L_{s}(t)$ begins at $\left[x_{0}, x_{n}\right]\left(y_{0}\right)$, where $\left[x_{0}, x_{n}\right]$ is the permutation of $B$ which interchanges $x_{0}$ and $x_{n}$ and fixes all other elements of $B$. Hence, if $L_{s}(t)$ and $L_{s}\left(t^{\prime}\right)$ have the same beginning, then so do $t$ and $t^{\prime}$, and the definitions of $L_{s}(t)$ and $L_{s}\left(t^{\prime}\right)$ fall into the same cases, i.e., $L_{s}(t)=t$ iff $L_{s}\left(t^{\prime}\right)=t^{\prime}, L_{s}(t)=s \odot t$ iff $L_{s}\left(t^{\prime}\right)=s \odot t^{\prime}$, and $L_{s}(t)=\check{s} \odot t$ iff $L_{s}\left(t^{\prime}\right)=\check{s} \odot t^{\prime}$. It follows easily that $L_{s}$ is one-to-one. Furthermore, $t, L_{\xi} L_{s}(t)$, and $L_{s} L_{\xi}(t)$ have the same beginning.

According to the definitions of $\odot$ and $L_{s}, t$ is always a final segment of $L_{s}(t)$. Consequently $L_{s}$ preserves $P_{1}, P_{2}$, and $P_{3}$, i.e., $L_{s}^{-1}\left|P_{1}\right| L_{s} \subseteq$ $P_{1}, L_{s}^{-1}\left|P_{2}\right| L_{s} \subseteq P_{2}$, and $L_{s}^{-1}\left|P_{3}\right| L_{s} \subseteq P_{3}$. So $L_{s}$ also preserves $Q$, i.e., $L_{s}^{-1}|Q| L_{s} \subseteq Q$.

To prove (i), first suppose $t^{\prime} \in l_{s}\left(t^{\mathfrak{B}}\right)$. Then there is some $t^{\prime \prime} \in t^{\mathfrak{B}}$ such that $t^{\prime} \in L_{s}\left(t^{\prime \prime}\right)^{\mathfrak{B}}$. Therefore $t^{\prime \prime} Q t$ and $t^{\prime} Q L_{s}\left(t^{\prime \prime}\right)$. Since $L_{s}$ preserves $Q$, we have $L_{s}\left(t^{\prime \prime}\right) Q L_{s}(t)$, so $t^{\prime} Q L_{s}(t)$, i.e., $t^{\prime} \in L_{s}(t)^{\mathfrak{B}}$. Thus $l_{s}\left(t^{\mathfrak{B}}\right) \subseteq L_{s}(t)^{\mathfrak{B}}$. The opposite inclusion holds trivially.

For the proof of (ii), first check that $L_{s} L_{s}(t)$ is either $t, \check{s} \odot s \odot t$, or $s \odot \check{s} \odot t$. We will show $L_{\dot{s}} L_{s}(t) \in t^{\mathfrak{B}}$ by considering three cases. Obviously $L_{\dot{s}} L_{s}(t) \in t^{\mathfrak{B}}$ if $L_{\dot{s}} L_{s}(t)=t$. Suppose $L_{\dot{s}} L_{s}(t)=\check{s} \odot s \odot t \neq t$. Then $|s|>1$ and $\check{s} \odot s \odot t \in D o P_{1}$. In fact, $\check{s} \odot s \odot t P_{1}^{|s|-1} t$, so $L_{s} L_{s}(t) \in t^{\mathfrak{B}}$. Similarly, if $L_{s} L_{s}(t)=s \odot \check{s} \odot t \neq t$, then $s \odot \check{s} \odot t P_{1}^{|s|-1} t$, so $L_{s} L_{s}(t) \in t^{\mathfrak{B}}$. From $L_{s} L_{s}(t) \in t^{\mathfrak{B}}$ and part (i) we get $t^{\mathfrak{B}}=\left(L_{s} L_{s}(t)\right)^{\mathfrak{B}}=l_{s}\left(L_{s}(t)^{\mathfrak{B}}\right)=l_{s} l_{s}\left(t^{\mathfrak{B}}\right)$, which shows one equality of (ii). The other equality holds similarly.

Finally, part (iii) follows from parts (i) and (ii).

By Lemma $11, \operatorname{Pm}(\mathfrak{B})$ is a group of permutations of the canonical base points. Hence the functions in $\operatorname{Pm}(\mathfrak{B})$ are called canonical permutations of $U(\mathfrak{B})$.

Lemma 12. Let $x \in B$. Then

(i) $\operatorname{Pm}(\mathfrak{B})$ preserves $\mathrm{R}_{x}^{\mathfrak{B}}$, and $\operatorname{Pm}(\mathfrak{B})$ acts transitively on $\mathrm{R}_{x}^{\mathfrak{B}}$.

(ii) $\mathrm{R}_{x}^{\mathfrak{B}} \in A t \mathfrak{R c} \mathfrak{B}$. 
Proof. By Lemma 9, (i) implies (ii). To prove (i), we first show that $\tilde{l}_{s} \mathrm{R}_{x}^{\mathfrak{B}} \subseteq \mathrm{R}_{x}^{\mathfrak{B}}$ for every $s \in P m(\mathfrak{B})$ using Lemma $11(\mathrm{i})$ :

$$
\begin{aligned}
\tilde{l}_{s} \mathrm{R}_{x}^{\mathfrak{B}} & =\left\{a \mid l_{s}: a \in\left\{\left\langle(t \kappa)^{\mathfrak{B}}: \kappa<\alpha\right\rangle: t \in \operatorname{Tr}(\mathfrak{B}), t \text { ends at } x\right\}\right\} \\
& =\left\{\left\langle(t \kappa)^{\mathfrak{B}}: \kappa<\alpha\right\rangle \mid l_{s}: t \in \operatorname{Tr}(\mathfrak{B}), t \text { ends at } x\right\} \\
& =\left\{\left\langle l_{s}\left((t \kappa)^{\mathfrak{B}}\right): \kappa<\alpha\right\rangle: t \in \operatorname{Tr}(\mathfrak{B}), t \text { ends at } x\right\} \\
& =\left\{\left\langle L_{s}(t \kappa)^{\mathfrak{B}}: \kappa<\alpha\right\rangle: t \in \operatorname{Tr}(\mathfrak{B}), t \text { ends at } x\right\} \\
& =\left\{\left\langle\left(L_{s}(t) \kappa\right)^{\mathfrak{B}}: \kappa<\alpha\right\rangle: t \in \operatorname{Tr}(\mathfrak{B}), t \text { ends at } x\right\} \\
& \subseteq \mathrm{R}_{x}^{\mathfrak{B}} .
\end{aligned}
$$

Since $l_{s}$ and $l_{s}$ are inverses, so are $\tilde{l}_{s}$ and $\tilde{l}_{\tilde{s}}$. Hence $\mathrm{R}_{x}^{\mathfrak{B}}=\tilde{l}_{\tilde{s}} \tilde{l}_{s} \mathbf{R}_{x}^{\mathfrak{B}} \subseteq \tilde{l}_{s} \mathrm{R}_{x}^{\mathfrak{B}} \subseteq \mathbf{R}_{x}^{\mathfrak{B}}$, so $\mathrm{R}_{x}^{\mathfrak{B}}$ is preserved by $l_{s}$.

To show that $\operatorname{Pm}(\mathfrak{B})$ acts transitively on $\mathrm{R}_{x}^{\mathfrak{B}}$, let us assume that $t$ and $t^{\prime}$ are trails which end at $x$. We wish to find a canonical permutation $l_{s}$ which maps $\left\langle(t \kappa)^{\mathfrak{B}}: \kappa<\alpha\right\rangle$ to $\left\langle\left(t^{\prime} \kappa\right)^{\mathfrak{B}}: \kappa<\alpha\right\rangle$. Let $s=t^{\prime} \odot \check{t}$. Note that $s$ is defined since $t^{\prime}$ ends where $t$ begins. Let $\kappa<\alpha$. Then $L_{s}(t \kappa)=t^{\prime} \odot \check{t} \odot t \kappa$. If $|t|=1$, then $t^{\prime} \odot \check{t} \odot t \kappa=t^{\prime} \kappa$, so $L_{s}(t \kappa) \in\left(t^{\prime} \kappa\right)^{\mathfrak{B}}$, and if $|t|>1$, then $t^{\prime} \odot \check{t} \odot t \kappa P_{1}^{|t|-1} t^{\prime} \kappa$, and again $L_{s}(t \kappa) \in\left(t^{\prime} \kappa\right)^{\mathfrak{B}}$. Hence $\left\langle(t \kappa)^{\mathfrak{B}}: \kappa<\alpha\right\rangle \mid l_{s}=$ $\left\langle l_{s}\left(t \kappa^{\mathfrak{B}}\right): \kappa<\alpha\right\rangle=\left\langle\left(L_{s}(t \kappa)\right)^{\mathfrak{B}}: \kappa<\alpha\right\rangle=\left\langle\left(t^{\prime} \kappa\right)^{\mathfrak{B}}: \kappa<\alpha\right\rangle$.

By Lemma 12(i), if $a \in \mathrm{R}_{x}^{\mathfrak{B}}$, then $\mathrm{R}_{x}^{\mathfrak{B}}=\{a \mid p: p \in P m(\mathfrak{B})\}$, i.e., every $\mathrm{R}_{x}^{\mathfrak{B}}$ is the orbit of a single sequence under the group $\operatorname{Pm}(\mathfrak{B})$.

Lemma 13. For every $x \in B$ and $\kappa, \lambda<\alpha, x \in E_{\kappa \lambda}$ iff $\mathrm{R}_{x}^{\mathfrak{B}} \subseteq \mathrm{D}_{\kappa \lambda}^{[V(\mathfrak{B})]}$.

Proof. If $\kappa=\lambda$ then the result holds by Definition 1(ii), so assume $\kappa \neq \lambda$ and $x \in E_{\kappa \lambda}$. Let $\left\langle(t \mu)^{\mathfrak{B}}: \mu<\alpha\right\rangle \in \mathrm{R}_{x}^{\mathfrak{B}}$. Then $t$ ends at $x$ and $t \kappa P_{3} t \lambda$, so $(t \kappa)^{\mathfrak{B}}=(t \lambda)^{\mathfrak{B}}$, which implies $\left\langle(t \mu)^{\mathfrak{B}}: \mu<\alpha\right\rangle \in \mathrm{D}_{\kappa \lambda}^{[V(\mathfrak{B})]}$.

Now assume $\mathrm{R}_{x}^{\mathfrak{B}} \subseteq \mathrm{D}_{\kappa \lambda}^{[V(\mathfrak{B})]}$. Let $t=\langle x, \kappa\rangle$. Then $\left\langle(t \mu)^{\mathfrak{B}}: \mu\langle\alpha\rangle \in \mathrm{R}_{x}^{\mathfrak{B}}\right.$, so $t Q t \lambda$. But $t$ and $t \lambda$ are not in the domain of $P_{5}$ since $|t|=|t \lambda|=1$, so $t P_{3} t \lambda$, i.e., $x \in E_{\kappa \lambda}$.

Lemma 14. If $x, y \in B, \mu<\alpha$, and $x T_{\mu} y$, then $\mathrm{R}_{x}^{\mathfrak{B}} \subseteq \mathrm{C}_{\mu}^{[V(\mathfrak{B})]} \mathrm{R}_{y}^{\mathfrak{B}}$.

Proof. If $x=y$, then $\mathrm{R}_{x}^{\mathfrak{B}}=\mathrm{R}_{y}^{\mathfrak{B}} \subseteq \mathrm{C}_{\mu}^{[V(\mathfrak{B})]} \mathrm{R}_{y}^{\mathfrak{B}}$, so we may assume $x \neq y$. Suppose $\left\langle(t \kappa)^{\mathfrak{B}}: \kappa<\alpha\right\rangle \in \mathrm{R}_{x}^{\mathfrak{B}}$. Let $t^{\prime}=t \odot\langle x, \mu, y, \mu\rangle$. Note that $\left\langle\left(t^{\prime} \kappa\right)^{\mathfrak{B}}\right.$ : $\kappa<\alpha\rangle \in \mathrm{R}_{y}^{\mathfrak{B}}$. If $\kappa \neq \mu$ then $t \kappa=(t \odot\langle x, \kappa\rangle) Q(t \odot\langle x, \mu, y, \kappa\rangle)=t^{\prime} \kappa$. So $(t \kappa)^{\mathfrak{B}}=\left(t^{\prime} \kappa\right)^{\mathfrak{B}}$ whenever $\kappa \neq \mu$, and therefore

$$
\left\langle(t \kappa)^{\mathfrak{B}}: \kappa<\alpha\right\rangle \in \mathrm{C}_{\mu}\left\{\left\langle(t \kappa)^{\mathfrak{B}}: \kappa<\alpha\right\rangle\right\} \cap V(\mathfrak{B}) \subseteq \mathrm{C}_{\mu}^{[V(\mathfrak{B})]} \mathrm{R}_{y}^{\mathfrak{B}} .
$$

Thus $\mathrm{R}_{x}^{\mathfrak{B}} \subseteq \mathrm{C}_{\mu}^{[V(\mathfrak{B})]} \mathrm{R}_{y}^{\mathfrak{B}}$ 


\section{$\S 3$ RePRESENTATION USING $\mathfrak{R} \mathfrak{A} \mathfrak{A} \mathfrak{A}$}

The next lemma uses terminology from [HMT71, 2.7.1, 2.7.32]. For every $\alpha$, $N C A_{\alpha}$ is the class of algebras which satisfy postulates $1.1 .1\left(C_{0}\right)-\left(C_{3}\right),\left(C_{5}\right)-$ $\left(\mathrm{C}_{7}\right)$ of [HMT71]. (See [N86] or [T].)

Lemma 15. Suppose $1 \leq \alpha$ and $\mathfrak{A}$ is a normal atomic $\alpha$-dimensional Boolean algebra with operators. Then $\mathfrak{A t} \mathfrak{A}$ is a suitable structure iff $\mathfrak{A} \in \mathbf{N C A}_{\alpha}$.

Proof. Imitate the proof of Theorem 2.7.40, [HMT71], noting that statement $(4)$, p. 456 , which corresponds to $\left(\mathrm{C}_{4}\right)$, is used only to obtain condition 2.7.40(ii), and conversely.

Definition 16. For every $\mathfrak{A} \in N C A_{\alpha}$, let $R^{\mathfrak{A}}$ be the mapping from $\mathfrak{A}$ into $\mathfrak{R} \mathfrak{A} \mathfrak{A} \mathfrak{A}$ defined by $\mathrm{R}^{\mathfrak{A}}(x)=\bigcup_{x \geq y \in A t \mathfrak{A}} \mathrm{R}_{y}^{\mathfrak{x} t \mathfrak{a}}$ for every $x \in A$.

Note that $\mathrm{R}^{\mathfrak{A}}(x)=\mathrm{R}_{x}^{\mathfrak{A} t \mathfrak{A}}$ whenever $x$ is an atom of $\mathfrak{A}$. The next lemma follows immediately from Lemmas 13 and 14 .

Lemma 17. If $\mathfrak{A} \in \mathrm{NCA}_{\alpha}$ and $\kappa, \lambda, \mu<\alpha$, then $\mathrm{R}^{\mathfrak{A}}\left(\mathrm{d}_{\kappa \lambda}\right)=\mathrm{D}_{\kappa \lambda}^{[V(\mathfrak{A} \mathfrak{A} \mathfrak{A})]}$ and $\mathrm{R}^{\mathfrak{A}}\left(\mathrm{c}_{\mu} x\right) \subseteq \mathrm{C}_{\mu}^{[V(\mathfrak{A} \mathfrak{A} \mathfrak{A})]}\left(\mathrm{R}^{\mathfrak{A}}(x)\right)$.

Theorem C. Suppose $2 \leq \alpha, \mathfrak{A} \in \mathrm{NA}_{\alpha}, \mathfrak{A}$ is complete, atomic, and satisfies $\mathrm{MGR}_{n}$ for $n=2,3$. Then $\mathfrak{A} \cong \mathfrak{R c} \mathfrak{A} \mathfrak{A} \in \mathrm{RICs}_{\alpha}$.

Proof. By Lemma $15, \mathfrak{A} \mathfrak{t A}$ is a suitable structure. By Lemma $12(\mathrm{ii}), \mathrm{R}^{\mathfrak{A}}$ maps $A t \mathfrak{A}$ onto $A t \mathfrak{R c} \mathfrak{A} \mathfrak{A} \mathfrak{A}$, so $\mathrm{R}^{\mathfrak{A}}$ is a Boolean isomorphism since $\mathfrak{A}$ is complete. By Lemma 17, we need only show that if $x, y \in A t \mathfrak{A}$ and $\mathrm{R}^{\mathfrak{A}}(x) \subseteq$ $\mathrm{C}_{\mu}^{[V(\mathfrak{A}+\mathfrak{a})]} \mathrm{R}^{\mathfrak{x} t \mathfrak{A}}(y)$, then $x \leq \mathrm{c}_{\mu} y$. To do this we imitate part of the proof of Theorem 1 in [AT]. Then

Whenever $\kappa, \lambda<\alpha, \kappa \neq \lambda$, and $x \in A$, let $\mathrm{t}_{\kappa}^{\kappa}(x)=x$ and $\mathrm{t}_{\lambda}^{\kappa}(x)=\mathrm{d}_{\kappa \lambda} \cdot \mathrm{c}_{\kappa} x$.

(1) $\mathrm{t}_{\lambda}^{\kappa}(x) \in A t \mathfrak{A}$ whenever $x \in A t \mathfrak{A}$,

(2) $\mathrm{t}_{\lambda}^{\kappa}(x)=x$ whenever $\mathrm{d}_{\kappa \lambda} \geq x \in A t \mathfrak{A}$,

(3) $\mathrm{t}_{\lambda}^{\kappa}(x)=\mathrm{t}_{\lambda}^{\kappa}(y)$ whenever $x \leq \mathrm{c}_{\kappa} y$ and $x, y \in A t \mathfrak{A}$.

These can be easily proved using [HMT71, 1.2.3, 1.2.5, 1.2.7, and 1.3.9]. The latter results apply to $\mathrm{NCA}_{\alpha}$, since their proofs rely only on postulates $\left(\mathrm{C}_{0}\right)-$ $\left(\mathrm{C}_{3}\right),\left(\mathrm{C}_{7}\right)$.

Let $\Omega=\bigcup_{n<\omega}{ }^{2(n+1)} \alpha$. If $\sigma=\left\langle\kappa_{0}, \lambda_{0}, \ldots, \kappa_{n}, \lambda_{n}\right\rangle \in \Omega$ then let $\sigma^{\mathfrak{A}}(x)=$ $\mathrm{t}_{\lambda_{0}}^{\kappa_{0}} \mathrm{t}_{\lambda_{1}}^{\kappa_{1}} \ldots \mathrm{t}_{\lambda_{n}}^{\kappa_{n}} x$ and let $\sigma^{\wedge}=\left[\kappa_{0} / \lambda_{0}\right]\left|\left[\kappa_{1} / \lambda_{1}\right]\right| \ldots \mid\left[\kappa_{n} / \lambda_{n}\right]$, where $[\kappa / \lambda]$ is the function mapping $\alpha$ to $\alpha$ which sends $\kappa$ to $\lambda$ and leaves every other $\mu<\alpha$ unchanged. Note that for all $\sigma, \tau \in \Omega,\left(\sigma^{\wedge} \tau\right)^{\wedge}=\sigma^{\wedge} \mid \tau^{\wedge}$, where $\sigma^{\wedge} \tau$ is the concatenation of $\sigma$ and $\tau$. By [AT, Lemma 1], we have

(4) if $\sigma, \tau \in \Omega$ and $\sigma^{\wedge}=\tau^{\wedge}$, then $\sigma^{\mathfrak{A}}(x)=\tau^{\mathfrak{A}}(x)$ for all $x \in A$.

The merry-go-round identities and $\left(\mathrm{C}_{4}^{*}\right)$ are needed only in the proof of (4). For every trail $t \in \operatorname{Tr}(\mathfrak{A} \mathfrak{A})$, let $[t]$ be the function from $\alpha$ to $\alpha$ such that for every $\kappa<\alpha,[t](\kappa)$ is the pointer of $P_{7}(t \kappa)$. We say that $t$ collapses on 
$X \subseteq \alpha$ if $\left|P_{7}(t \kappa)\right|=1$ for every $\kappa \in X$. We prove the next statement by induction on $|t|$.

(5) Assume $t=\left\langle x_{0}, \kappa_{0}, \ldots, x_{n}, \kappa_{n}\right\rangle \in \operatorname{Tr}(\mathfrak{A} \mathfrak{A} \mathfrak{A}), \sigma, \tau \in \Omega, t$ collapses on $R g \tau^{\wedge}$, and $\sigma^{\wedge}\left|\left[\left\langle x_{0}, \kappa_{0}\right\rangle\right]=\tau^{\wedge}\right|[t]$. Then $\sigma^{\mathfrak{A}}\left(x_{0}\right)=\tau^{\mathfrak{A}}\left(x_{n}\right)$.

Suppose first that $|t|=1$. Then $x_{0}=x_{n}$ and $t=\left\langle x_{0}, \kappa_{0}\right\rangle$. For any $\kappa<\alpha$, $P_{7}(t \kappa)=P_{7}\left(\left\langle x_{0}, k\right\rangle\right)=\left\langle x_{0}, \lambda\right\rangle=t \lambda$ for some $\lambda \leq \kappa$, i.e., $\lambda=[t](\kappa)$. But $t \lambda$ is reduced, so $P_{7}(t \lambda)=t \lambda$, hence $\lambda=[t](\lambda)$. This shows that $[t] \mid[t]=[t]$. To prove (5) in this case, we assume $\sigma, \tau \in \Omega$ and $\sigma^{\wedge}\left|[t]=\tau^{\wedge}\right|[t]$. (The assumption about $t$ collapsing on $\operatorname{Rg} \tau^{\wedge}$ is vacuous, since $t$ collapses on $\alpha$ by virtue of its length.)

If $I d_{\alpha}=[t]$ then the result follows by (4).

Assume $I d_{\alpha} \neq[t]$. Since $\sigma$ and $\tau$ are finite sequences, $\sigma^{\wedge}$ and $\tau^{\wedge}$ can move only finitely many ordinals. The following set of pairs is therefore finite: $M=\left\{\left\langle\sigma^{\wedge}(\kappa),[t]\left(\sigma^{\wedge}(\kappa)\right)\right\rangle: \sigma^{\wedge}(\kappa) \neq \kappa<\alpha\right\} \cup\left\{\left\langle\tau^{\wedge}(\kappa),[t]\left(\tau^{\wedge}(\kappa)\right)\right\rangle: \tau^{\wedge}(\kappa) \neq\right.$ $\kappa<\alpha\}$. Since $M$ is finite, there is some $\delta \in \Omega$ which is the result of concatenating all the pairs of $M$ in some order. From $[t] \mid[t]=[t]$ and the assumption $\sigma^{\wedge}\left|[t]=\tau^{\wedge}\right|[t]$ it is easy to show that $\left(\sigma^{\wedge} \delta\right)^{\wedge}=\left(\tau^{\wedge} \delta\right)^{\wedge}$, so by (4), $\sigma^{\mathfrak{A}}\left(\delta^{\mathfrak{A}}\left(x_{0}\right)\right)=\left(\sigma^{-} \delta\right)^{\mathfrak{A}}\left(x_{0}\right)=\left(\tau^{\frown} \delta\right)^{\mathfrak{A}}\left(x_{0}\right)=\tau^{\mathfrak{A}}\left(\delta^{\mathfrak{A}}\left(x_{0}\right)\right)$. Since $|t|=1$, if $\langle\kappa, \lambda\rangle \in M$, then $t \kappa P_{3} t \lambda$ and $x_{0} \leq \mathrm{d}_{\kappa \lambda}$. It is therefore easy to show by induction, using (2), that $\delta^{\mathfrak{A}}\left(x_{0}\right)=x_{0}$. Hence $\sigma^{\mathfrak{A}}\left(x_{0}\right)=\tau^{\mathfrak{A}}\left(x_{0}\right)$, as desired.

For the inductive case, let $n \geq 1$, and assume that (5) holds for all trails of length no more than $n$. Let $t=\left\langle x_{0}, \kappa_{0}, \ldots, x_{n-1}, \kappa_{n-1}, x_{n}, \kappa_{n}\right\rangle$, and set $t^{\prime}=$ $\left\langle x_{0}, \kappa_{0}, \ldots, x_{n-1}, \kappa_{n-1}\right\rangle$. Therefore $|t|=\left|t^{\prime}\right|+1=n+1$. Assume $\sigma, \tau \in \Omega$, $t$ collapses on $\operatorname{Rg} \tau^{\wedge}$, and $\sigma^{\wedge}\left|\left[\left\langle x_{0}, \kappa_{0}\right\rangle\right]=\tau^{\wedge}\right|[t]$. We wish to prove $\sigma^{\mathfrak{A}}\left(x_{0}\right)=$ $\tau^{\mathfrak{A}}\left(x_{n}\right)$.

Suppose that $t \in D o P_{1}$. Choose $t^{\prime \prime} \in \operatorname{Tr}(\mathfrak{A t A})$ so that $t P_{1} t^{\prime \prime}$. Obviously $\left|t^{\prime \prime}\right|=|t|-2=n-1<n$, so we may apply (5) to $t^{\prime \prime}$. (This case cannot occur when $n<3$.) Notice that $t^{\prime \prime}$ begins at $x_{0}$ and ends at $x_{n}$. By the definition of $P_{1}$, we clearly have $t \kappa P_{1} t^{\prime \prime} \kappa$ for every $\kappa<\alpha$. Consequently $P_{7}(t \kappa)=P_{7}\left(t^{\prime \prime} \kappa\right)$ for all $\kappa<\alpha$. It follows that $t^{\prime \prime}$ collapses on $\operatorname{Rg} \tau^{\wedge}$ since $t$ is assumed to do so. It also follows that $[t]=\left[t^{\prime \prime}\right]$, and hence $\sigma^{\wedge}\left|\left[\left\langle x_{0}, \kappa_{0}\right\rangle\right]=\tau^{\wedge}\right|[t]=\tau^{\wedge} \mid\left[t^{\prime \prime}\right]$. We may therefore apply the inductive hypothesis to $t^{\prime \prime}, \sigma$ and $\tau$, obtaining $\sigma^{\mathfrak{A}}\left(x_{0}\right)=\tau^{\mathfrak{A}}\left(x_{n}\right)$.

This completes the proof of (5) in case $t \in D o P_{1}$. Therefore assume $t \notin$ Do $P_{1}$. We consider two cases.

Suppose there is some $\lambda<\alpha$ such that $\lambda \neq \kappa_{n-1}$ and $x_{n} \leq \mathrm{d}_{\kappa_{n-1} \lambda}$. Set $v=$ $\tau^{\wedge}\left\langle\kappa_{n-1}, \lambda\right\rangle$. Notice that $v^{\wedge}=\left(\tau^{\wedge}\left\langle\kappa_{n-1}, \lambda\right\rangle\right)^{\wedge}=\tau^{\wedge}\left|\left\langle\kappa_{n-1}, \lambda\right\rangle^{\wedge}=\tau^{\wedge}\right|\left[\kappa_{n-1} / \lambda\right]$, so $R g v^{\wedge} \subseteq R g \tau^{\wedge} \sim\left\{\kappa_{n-1}\right\}$.

Next we show $t^{\prime}$ collapses on $R g v$. Let $\mu \in R g v^{\wedge}$. Then $\mu \in R g \tau^{\wedge}$, so $\left.\mid P_{7}(t \mu)\right) \mid=1$ since $t$ collapses on $R g \tau^{\wedge}$. Also, $\mu \neq \kappa_{n-1}$, so $t \mu P_{2} t^{\prime} \mu$, hence 
$P_{7}(t \mu)=P_{7}\left(t^{\prime} \mu\right)$, which implies $1=\left|P_{7}(t \mu)\right|=\left|P_{7}\left(t^{\prime} \mu\right)\right|$. Thus $t^{\prime}$ collapses on $R g v$.

Next we show $[t]=\left[\kappa_{n-1} / \lambda\right]\left[\left[t^{\prime}\right]\right.$. Since $\lambda \neq \kappa_{n-1}$, we have $t \kappa_{n-1}\left(P_{4} \cup\right.$ $\left.P_{4}^{-1}\right) t \lambda P_{2} t^{\prime} \lambda$, so $P_{7}\left(t \kappa_{n-1}\right)=P_{7}(t \lambda)=P_{7}\left(t^{\prime} \lambda\right)$, hence $[t]\left(\kappa_{n-1}\right)=[t](\lambda)=$ $\left[t^{\prime}\right](\lambda)=\left(\left[\kappa_{n-1} / \lambda\right] \mid\left[t^{\prime}\right]\right)\left(\kappa_{n-1}\right)$. On the other hand, if $\mu \neq \kappa_{n-1}$, then $t \mu P_{2} t^{\prime} \mu$, so $P_{7}(t \mu)=P_{7}\left(t^{\prime} \mu\right)$, hence $[t](\mu)=\left[t^{\prime}\right](\mu)=\left(\left[\kappa_{n-1} / \lambda\right] \mid\left[t^{\prime}\right]\right)(\mu)$. Thus $[t]=$ $\left[\kappa_{n-1} / \lambda\right] \mid\left[t^{\prime}\right]$. By assumption, $\sigma^{\wedge}\left|\left[\left\langle x_{0}, \kappa_{0}\right\rangle\right]=\tau^{\wedge}\right|[t]$, but $\tau^{\wedge} \mid[t]=$ $\tau^{\wedge}\left|\left[\kappa_{n-1} / \lambda\right]\right|\left[t^{\prime}\right]=v^{\wedge} \mid\left[t^{\prime}\right]$, so $\sigma^{\wedge}\left|\left[\left\langle x_{0}, \kappa_{0}\right\rangle\right]=v^{\wedge}\right|\left[t^{\prime}\right]$. We may apply our inductive hypothesis (5) to $t^{\prime}, \sigma$, and $v$, obtaining $\sigma^{\mathfrak{A}}\left(x_{0}\right)=v^{\mathfrak{A}}\left(x_{n-1}\right)$. However, $v^{\mathfrak{A}}\left(x_{n-1}\right)=\left(\tau^{\sim}\left\langle\kappa_{n-1}, \lambda\right\rangle\right)^{\mathfrak{A}}\left(x_{n-1}\right)=\tau^{\mathfrak{A}}\left(\mathrm{t}_{\lambda}^{\kappa_{n-1}} x_{n-1}\right)$ by the relevant definitions. Furthermore, we have $x_{n-1} \leq \mathrm{c}_{\kappa_{n-1}} x_{n}$ and $x_{n} \leq \mathrm{d}_{\kappa_{n-1} \lambda}$, so $\mathrm{t}_{\lambda}^{\kappa_{n-1}} x_{n-1}=$ $\mathrm{t}_{\lambda}^{x_{n-1}} x_{n}=x_{n}$ by (2) and (3). Therefore, $\sigma^{\mathfrak{A}}\left(x_{0}\right)=\tau^{\mathfrak{A}}\left(x_{n}\right)$. This completes the proof of (5) in the first case.

Suppose there is no $\lambda<\alpha$ such that $\lambda \neq \kappa_{n-1}$ and $x_{n} \leq \mathrm{d}_{\kappa_{n-1} \lambda}$. We are assuming $t \notin D o P_{1}$, and the assumptions for this case imply that $t \kappa_{n-1} \notin$ Do $\left(P_{4} \cup P_{4}^{-1} \cup P_{2}\right)$, so $t \kappa_{n-1}$ is reduced. Therefore $P_{7}\left(t \kappa_{n-1}\right)=t \kappa_{n-1}$. Now $\left|P_{7}\left(t \kappa_{n-1}\right)\right|=n+1>1$ and $t$ collapses on $R g \tau^{\wedge}$, so $\kappa_{n-1} \notin R g \tau^{\wedge}$. Choose any $\lambda<\alpha$ such that $\lambda \neq \kappa_{n-1}$. This is possible since $\alpha \geq 2$. Set $v=$ $\tau^{\curlyvee}\left\langle\kappa_{n-1}, \lambda\right\rangle$. As above, we have $v^{\wedge}=\tau^{\wedge} \mid\left[\kappa_{n-1} / \lambda\right]$, but this time $\kappa_{n-1} \notin$ $R g \tau^{\wedge}$, so $v^{\wedge}=\tau^{\wedge}$. If $\mu \neq \kappa_{n-1}$ (in particular, if $\mu \in R g v^{\wedge}=R g \tau^{\wedge}$ ) then $t \mu P_{2} t^{\prime} \mu$, so $P_{7}(t \mu)=P_{7}\left(t^{\prime} \mu\right)$ and $[t](\mu)=\left[t^{\prime}\right](\mu)=\left(\left[\kappa_{n-1} / \lambda\right] \mid\left[t^{\prime}\right]\right)(\mu)$. Now $t$ collapses on $R g \tau^{\wedge}$, so $t^{\prime}$ collapses on $R g v^{\wedge}$. It is possible that $[t]$ and $\left[\kappa_{n-1} / \lambda\right] \mid\left[t^{\prime}\right]$ differ on $\kappa_{n-1}$. However, since $\kappa_{n-1} \notin R g \tau^{\wedge}$ we have $\tau^{\wedge}=\tau^{\wedge} \mid\left[\kappa_{n-1} / \lambda\right]$, hence $\tau^{\wedge}\left|[t]=\tau^{\wedge}\right|\left[\kappa_{n-1} / \lambda\right]\left|\left[t^{\prime}\right]=v^{\wedge}\right|\left[t^{\prime}\right]$. By assumption, $\sigma^{\wedge}\left|\left[\left\langle x_{0}, \kappa_{0}\right\rangle\right]=\tau^{\wedge}\right|[t]$, so $\sigma^{\wedge}\left|\left[\left\langle x_{0}, \kappa_{0}\right\rangle\right]=v^{\wedge}\right|\left[t^{\prime}\right]$.

We may now apply the inductive hypothesis to $t^{\prime}, \sigma$, and $\tau$, obtaining $\sigma^{\mathfrak{A}}\left(x_{0}\right)=v^{\mathfrak{A}}\left(x_{n-1}\right)$. Since $x_{n-1} \leq \mathrm{c}_{\kappa_{n-1}} x_{n}$, we have $\mathrm{t}_{\lambda}^{\kappa_{n-1}} x_{n-1}=\mathrm{t}_{\lambda}^{\kappa_{n-1}} x_{n}$ by (3). Therefore $\sigma^{\mathfrak{A}}\left(x_{0}\right)=v^{\mathfrak{A}}\left(x_{n-1}\right)=\tau^{\mathfrak{A}}\left(\mathrm{t}_{\lambda}^{\kappa_{n-1}} x_{n-1}\right)=\tau^{\mathfrak{A}}\left(\mathrm{t}_{\lambda}^{\kappa_{n-1}} x_{n}\right)=v^{\mathfrak{A}}\left(x_{n}\right)$. But we also have $\tau^{\wedge}\left|\left[\left\langle x_{n}, \kappa_{n}\right\rangle\right]=v^{\wedge}\right|\left[\left\langle x_{n}, \kappa_{n}\right\rangle\right]$ since $\tau^{\wedge}=v^{\wedge}$, and trails of length 1 collapse on $\alpha$, so by the inductive hypothesis, applied to $\left[\left\langle x_{n}, \kappa_{n}\right\rangle\right], \tau$, and $v$, we get $\tau^{\mathfrak{A}}\left(x_{n}\right)=v^{\mathfrak{A}}\left(x_{n}\right)$. Hence $\sigma^{\mathfrak{A}}\left(x_{0}\right)=\tau^{\mathfrak{A}}\left(x_{n}\right)$.

This completes the proof of (5).

To finish the proof of the theorem, suppose $x, y \in A t \mathfrak{A}$ and $\mathrm{R}^{\mathfrak{A}}(x) \subseteq$ $\mathrm{C}_{\mu}^{[V(\mathfrak{A t a t})]} \mathrm{R}^{\mathfrak{A}}(y)$. We wish to show $x \leq \mathrm{c}_{\mu} y$. Since $x \leq \mathrm{c}_{\mu} x$, we may assume $x \neq y$. Let $s=\langle x, \mu\rangle$. Then $\left\langle(s \kappa)^{\mathfrak{A} \mathfrak{A} \mathfrak{A}}: \kappa<\alpha\right\rangle \in \mathrm{R}_{x}^{\mathfrak{A}}$, so, by our hypothesis, there is some reduced trail $t \in \operatorname{Tr}(\mathfrak{A} \mathfrak{A} \mathfrak{A})$ such that $\left\langle(t \kappa)^{\mathfrak{A} \mathfrak{A}}: \kappa<\alpha\right\rangle \in \mathrm{R}_{y}^{\mathfrak{A}}$ and s $Q$ t $\kappa$ whenever $\mu \neq \kappa<\alpha$. Choose $\kappa<\alpha$ so that $\mu \neq \kappa$. This is possible since $\alpha \geq 2$. It follows from $s \kappa Q t \kappa$ that $s$ and $t$ both begin at $x$. Note that 
$t$ ends at $y, t$ collapses on $\operatorname{Rg}[\mu / \kappa]$ and $[\mu / \kappa]|[s]=[\mu / \kappa]|[t]$. Therefore, by (5), $\mathrm{t}_{\kappa}^{\mu} x=\mathrm{t}_{\kappa}^{\mu} y$, which implies $x \leq c_{\mu} y$.

\section{REFERENCES}

[AT] H. Andréka and R. J. Thompson, Stone type representation theorem for algebras of relations of higher rank, Trans. Amer. Math. Soc. (to appear).

[HR75] L. Henkin and D. Resek, Relativization of cylindric algebras, Fund. Math. 82 (1975), 363-383.

[HMT71] L. Henkin, J. D. Monk, and A. Tarski, Cylindric Algebras, Part I, North-Holland Publishing Company, Amsterdam-London, 1971. (Also, Studies in logic and the foundations of mathematics, 64).

[HMT85] _ _, Cylindric Algebras, Part II, Elsevier Science Publishers B.V., North-Holland, Amsterdam-New York-Oxford, 1985. (Also, Studies in logic and the foundations of mathematics, 115).

[M82] R. D. Maddux, Some varieties containing relation algebras, Trans. Amer. Math. Soc. 272 (1982), 501-526.

[M89] _ Nonfinite axiomatizability results for cylindric and relation algebras, J. Symbolic Logic 54 (3) (to appear).

[N86] I. Németi, Free Algebras and Decidability in Algebraic Logic, Doctoral dissertation, Hungarian Academy of Sciences, Budapest, 1986.

[R75] D. Resek, Some results on relativized cylindric algebras, Doctoral dissertation, University of California, Berkeley, 1975, iii +290.

[T] R. J. Thompson, Noncommutative cylindric algebras and relativizations of cylindric algebras, preprint, 1987.

Department of Mathematics, Iowa State University, Ames, Iowa 50011 